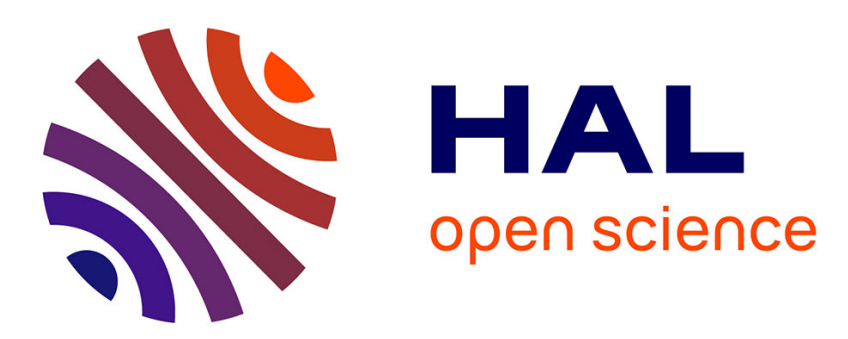

\title{
Thermomechanical effects accompanying the localized necking of semi-crystalline polymers
}

Bertrand Wattrisse, Jean-Michel Muracciole, André Chrysochoos

\section{To cite this version:}

Bertrand Wattrisse, Jean-Michel Muracciole, André Chrysochoos. Thermomechanical effects accompanying the localized necking of semi-crystalline polymers. International Journal of Thermal Sciences, 2002, 41 (5), pp.422-427. 10.1016/S1290-0729(02)01334-0 . hal-03350348

\section{HAL Id: hal-03350348 \\ https://hal.science/hal-03350348}

Submitted on 21 Sep 2021

HAL is a multi-disciplinary open access archive for the deposit and dissemination of scientific research documents, whether they are published or not. The documents may come from teaching and research institutions in France or abroad, or from public or private research centers.
L'archive ouverte pluridisciplinaire HAL, est destinée au dépôt et à la diffusion de documents scientifiques de niveau recherche, publiés ou non, émanant des établissements d'enseignement et de recherche français ou étrangers, des laboratoires publics ou privés. 


\title{
Thermomechanical effects accompanying the localized necking of semi-crystalline polymers
}

\section{Effets thermomécaniques accompagnant la striction localisée de polymères semi-cristallins}

\author{
Bertrand Wattrisse, Jean-Michel Muracciole, André Chrysochoos* \\ Université Montpellier II, Laboratoire de Mécanique et de Génie Civil, Place E. Bataillon, C.C. 081, Bât. 13, 34095 Montpellier cedex 5, France
}

\begin{abstract}
This paper presents an application of quantitative infrared thermography to the analysis of the mechanical behavior of materials. We particularly focus on the thermomechanical behavior of a semi-crystalline polymer below its glass transition temperature. During a quasistatic tensile test, the temperature distribution at the surface of a thin flat sample is recorded by an infrared camera. Using a local expression of the heat balance, the distribution of heat sources is derived from thermal measurements. These calorimetric data are then correlated with strain and stress fields. Indeed, a second optical device gives displacement fields using correlation methods on speckle images. Strain and strain-rate are derived from displacement data by numerical differentiation. The load-displacement curve shows three stages: first the load increases with the stretching, then a significant softening of the sample occurs until it flows at constant load. Both calorimetric and kinematic measurements indicate that the sample softening is associated with a progressive localization of heat sources and strain-rates, while the loading plateau corresponds with a regular expansion of the neck. A local construction of stress-strain diagram is then proposed. Several stress-strain curves are finally analyzed taking into account the loading stage characteristics and the expansion mode of the necking region.
\end{abstract}

Keywords: Quantitative infrared thermography; Material behavior; Thermomechanical couplings; Localization; Necking

\section{Introduction}

This paper deals with the behavior of a semi-crystalline thermoplastic polymer submitted to a quasi-static monotone tensile test. A classical way for describing the macroscopic behavior of material consists in considering it not only as a continuous medium but also as a thermodynamic system. Thermomechanics of Solid Materials aims at presenting the constitutive equations within a framework which adopts at the same time the tools of Mechanics, while seeking to translate the macroscopic consequences of the microstructural phenomena through state variables $[1,2]$. In such a

\footnotetext{
* Correspondence and reprints.

E-mail addresses: wattrisse@1mgc.univ-montp2.fr (B. Wattrisse), muracciole@1mgc.univ-montp2.fr (J.-M. Muracciole), chrysochoos@1mgc.univ-montp2.fr (A. Chrysochoos).
}

context, the choice of the temperature and of a strain tensor as state variables seems to be legitimate. It is then of great importance to determine their evolutions during particular tests to establish and/or identify the constitutive equations.

To get this thermomechanical information, we developed two independent complementary imaging techniques.

As other authors [3-6], infrared techniques were used to monitor the temperature at the surface of the sample. The originality of our approach was to use the infrared data to estimate the heat sources involved in the straining via a locally depth-wise averaged heat equation $[7,8]$. The lefthand member of this equation was consequently written as a partial derivative operator applied to the temperature while the different heat sources due to energy dissipation and to thermomechanical couplings were grouped in the righthand member. Information on the nature of the heat sources 


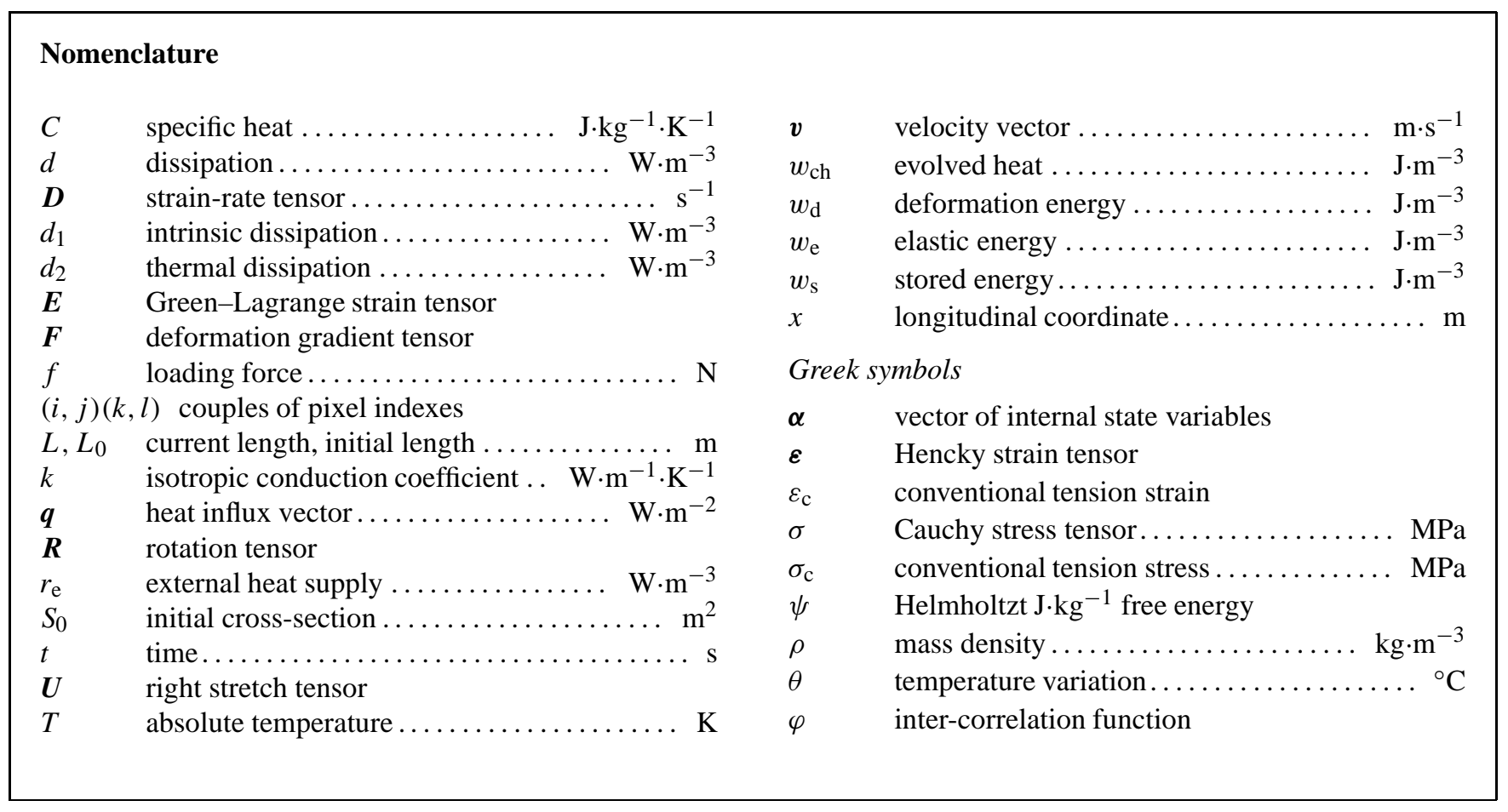

induced by the deformation process is very important for modeling the material behavior. Indeed, the coupling sources are linked to the state equations while the intrinsic dissipation is related to the evolution equations, both groups representing the set of constitutive equations.

The second imaging method was based on digital image correlation. The surface of the sample being speckled with white paint, these speckles play the role of local optical signatures that can be tracked during the deformation by using correlation methods. These techniques have been used for a long time and provide whole-field in-plane displacement data for plane structure $[9,10]$. To calculate the third displacement component, we assumed material incompressibility and depth-wise homogeneity of the strain field [11]. The strain and strain-rate tensors were then derived by differentiation. The tensile stress distribution was also computed assuming its uniformity within each crosssection of the sample.

This paper is composed as follows: in Section 2, the energy balance form and the heat equation are briefly reviewed. The Section 3 mentions the main characteristics of the experimental set-up and gives an outline of the numerical tools developed for image processing. The Section 4 focuses on the most representative results obtained on a semi-crystalline thermoplastic polymer stretched at room temperature. In particular, the similarity of the localization effects, in terms of heat source and strain-rate, is underlined and put forward to interpret the non-monotone mechanical response of the sample during the tensile test.

\section{Thermomechanical background}

The framework of Irreversible Thermodynamics is used. It assumes the local state axiom [12,13]. Classically, we characterize the equilibrium of a volume element by the absolute temperature $T$, a strain tensor $\boldsymbol{\varepsilon}$, and a $n$ scalar components vector $\boldsymbol{\alpha}$ complete the description of the equilibrium state.

If $\psi$ and $s$ denote respectively the specific Helmholtz free energy and the specific entropy, the Clausius-Duhem inequality, derived form of the second principle of thermodynamics, defines the dissipation $d$ as

$d=\sigma: D-\rho \psi_{\varepsilon}: \dot{\varepsilon}-\rho \psi_{\alpha} \cdot \dot{\alpha}-\frac{q}{T} \cdot \operatorname{grad} T \geqslant 0$

where $\boldsymbol{\sigma}$ is the Cauchy stress tensor, $\boldsymbol{D}$ the Eulerian strain rate tensor, $\rho$ the mass density, $\boldsymbol{q}$ the heat influx vector. The dot on letter stands for the material time derivative. The equality $d=0$ then characterizes reversible thermodynamic processes. Classically, [1,2], $d$ is split into two terms $d_{1}$ and $d_{2}$ where $d_{1}$ is the intrinsic (mechanical) dissipation while $d_{2}$ symbolizes the thermal dissipation-both supposed to be separately positive. They are, respectively, defined by

$d_{1}=\boldsymbol{\sigma}: \boldsymbol{D}-\rho \boldsymbol{\psi}_{\varepsilon}: \dot{\boldsymbol{\varepsilon}}-\rho \boldsymbol{\psi}_{\boldsymbol{\alpha}} \cdot \dot{\boldsymbol{\alpha}} \geqslant 0$

$d_{2}=-\frac{q}{T} \cdot \operatorname{grad} T \geqslant 0$

Per unit volume, the intrinsic dissipation $d_{1}$ is the difference between the rate of deformation work $w_{\text {def }}^{\bullet}=\sigma: \boldsymbol{D}$ and the rates of elastic $\left(w_{\mathrm{e}}^{\bullet}\right)$ and stored $\left(w_{\mathrm{s}}^{\bullet}\right)$ energy. The symbol ( $)^{\bullet}$ means that the time variation of () is path-dependent.

Deduced from both principles of thermodynamics, the local heat conduction equation reads 


$$
\begin{aligned}
& \rho C \dot{T}+\operatorname{div} \boldsymbol{q} \\
& \quad=d_{1}+\rho T \boldsymbol{\psi}_{\boldsymbol{T} \boldsymbol{\varepsilon}}: \dot{\boldsymbol{\varepsilon}}+\rho T \boldsymbol{\psi}_{\boldsymbol{T} \boldsymbol{\alpha}} \cdot \dot{\boldsymbol{\alpha}}+r_{\mathrm{e}}=w_{\mathrm{ch}}^{\bullet}
\end{aligned}
$$

where $C$ denotes the specific heat capacity while $r_{\mathrm{e}}$ symbolizes the external heat supply. The intrinsic dissipation $d_{1}$ and the thermomechanical coupling terms $\rho T \boldsymbol{\psi}_{T \varepsilon}: \dot{\varepsilon}$ and $\rho T \psi_{T \alpha} \cdot \dot{\alpha}$ have been grouped in the right-hand member of Eq. (4). For some materials, the term $\rho T \psi_{T \varepsilon}: \dot{\varepsilon}$ may represent the volume heat source due to thermoelastic couplings.

Taking into account a homogeneous isotropic conduction of heat $(\boldsymbol{q}=-k \operatorname{grad} T)$, we underline that the left side of Eq. (4) is a partial derivative operator applied to temperature. Its estimate leads to a local determination of the overall heat source.

\section{Experimental arrangement}

The experimental set-up involves a $100 \mathrm{kN}$ tensioncompression, servo-mechanic testing machine. The cameras are placed in the front of the testing machine, the axis of the lenses being perpendicular to the sample surface. In this work, we used separately either the infrared camera or the CCD video camera. In other words, thermography analysis and digital image correlation were performed by using a same testing machine in the same testing conditions but on different samples.

\subsection{Infrared image processing}

The heat equation (4) offers, under certain conditions, a direct link between the temperature variations and volume heat sources. In the framework of our experiments this equation can be simplified as follows [3]:

$\rho C\left(\frac{\partial \theta}{\partial t}+\boldsymbol{v} \cdot \operatorname{grad} \theta+\frac{\theta}{\tau_{\mathrm{th}}}\right)-k \frac{\partial^{2} \theta}{\partial x^{2}}=w_{\mathrm{ch}}^{\bullet}$

where $v$ is the velocity vector, $\tau_{\text {th }}$ is a time constant characterizing the lateral heat losses and $-k \partial^{2} \theta / \partial x^{2}$ represents the longitudinal heat losses, $O x$ being the pulling direction. The variable $\theta$ denotes the temperature variation between current and initial states. If the convective terms $\boldsymbol{v} \cdot \operatorname{grad} \theta$ are negligible, the volume heat source $w_{\mathrm{ch}}^{\bullet}$ is directly derived from an estimate of the left-hand member of Eq. (5). Naturally, this approximation can be disputable for polymers having a weak diffusivity and for deformation processes giving rise to localization.

To estimate the left-hand side of Eq. (5), the thermal noise is to be reduced before any differentiation. In this work, this estimate was performed by using a local least-squares fitting. The noisy discrete temperature field was locally fitted by parabolic function, corresponding to piecewise constant heat losses on a chosen neighborhood of each pixel of the thermal image. Of course, the size of the approximation zone affects the data smoothing, and thus influences the efficiency of the filtering. Tests were necessary to get a good compromise between speed and accuracy. We chose $15 \times 15$ square pixels as approximation zone; this size takes into account the signal-to-noise ratio, the space resolution of data and the degree of heterogeneity we wish to detect. We chose to implement a centered numerical scheme to fit the temperature data. To avoid zero-padding at the boundary of images, an image extension was made before the data fitting step. The parabolic approximation function was adjusted by using the temperature data available on one side of the boundary. The image extension was then computed by using this function.

\subsection{Kinematic measurements}

The digital speckle images were recorded by a C.C.D. camera. The lens axis of the camera was kept fixed with reference to the frame of the testing machine and perpendicular to the surface of the sample. The image processing was realized after the test itself and was split into two steps. First, the displacement field was estimated at points $M$ of a chosen virtual grid defined in the reference configuration. The displacement at $M$ is naturally decomposed into 2 in-plane components and 1 out of plane component. The two first can be computed by a correlation technique. Between two images $I_{1}$ and $I_{2}$, separated by a small strain increment, the displacement (in pixels) at $M$ located at pixel $(i, j)$ in the initial image, is given by the couple $(k, l)$ maximizing the intercorrelation function $\varphi$ defined by [9]:

$$
\begin{aligned}
& \begin{array}{c}
\varphi(k, l) \\
(k, l) \in\left[\frac{-R Z}{2}, \frac{R Z}{2}\right]
\end{array} \\
& =\frac{\sum_{i=-C Z / 2 \sum_{j=-C Z / 2}^{C Z / 2} I_{1}(i, j) \cdot I_{2}(i+k, j+l)}^{C Z / 2}}{\sqrt{\sum_{i=-C Z / 2}^{C Z / 2} \sum_{j=-C Z / 2}^{C Z / 2} I_{1}^{2}(i, j)}} \\
& \times \frac{1}{\sqrt{\sum_{i=-C Z / 2}^{C Z / 2} \sum_{j=-C Z / 2}^{C Z / 2} I_{2}^{2}(i+k, j+l)}}
\end{aligned}
$$

where:

- $C Z$ is the number of pixels defining the correlation zone (i.e., $M$ 's neighborhood corresponding to a local optical signature of point $M$ );

- $R Z$ is the number of pixels defining the research zone.

To reach a sub-pixel resolution, a local quadratic interpolation was performed in the vicinity of the discrete maximum of $\varphi$ as proposed in [10]. The third displacement component could be computed by assuming material incompressibility.

The strain (or strain-rate) field was then derived from the displacements by space (and time) differentiation. To get a good accuracy, the displacement field had to be filtered too. Once again, we adopted a local least-squares fitting to process the displacement data: a linear regression was applied to each component of the displacement. 


\subsection{Checking of image processing}

The performances of both image processing were tested on analytic and experimental cases corresponding to various heat source distributions and different strain patterns [8, 11]. The validity check of the out of plane displacement computation was performed by comparing the thickness evolution of a steel sample after a tensile test, given by a three-axis measurement machine with the one given by the computation [11]. Using standard parameters, the accuracy on temperature variations was around $10^{-2}{ }^{\circ} \mathrm{C}$, while for displacement calculations, the resolution was about $5 \times 10^{-2}$ pixel, which corresponds with $5 \times 10^{-4}$ in terms of strain.

\section{Results}

The thermomechanical behavior of a semi-crystalline thermoplastic polymer (polyamide) was studied for uniaxial monotone loading tests performed on ISO R527 samples.

\subsection{Presentation mode}

Imaging techniques use and provide a lot of information. In this work, they give access to time evolution of scalar fields (kinetic energy, temperature, heat source), but also fields of vectorial nature (displacement, velocity, acceleration) and even tensor fields (strain, strain-rate). Because heat source distributions and the strain-rate fields were found to be rather homogeneous along the sample width, we were led to propose the following representation of data: for concision's sake we plotted temporal evolution of scalar profiles captured along the longitudinal axis of the sample. Further to simplify the data interpretation, contour plots were chosen for quick visualization of the phenomenon patterns. Spacetime charts were then constructed with the abscissa axis (top) representing the time and the ordinate axis (right) being the longitudinal sample axis. The conventional stress-strain diagram was superimposed to give the reader a familiar landmark to link the local pattern of the measurements to the loading state of the sample (left, bottom). For such a diagram we used the conventional stress and strain which are classically defined by:

$\sigma_{\mathrm{c}}=\frac{f}{S_{0}}$

$\varepsilon_{\mathrm{c}}=\frac{L-L_{0}}{L_{0}}$

where $f$ is the loading force, $S_{0}, L_{0}$ and $L$ being the initial cross-section, the initial and current lengths of the test section, respectively. Of course, classical strain and strainrate tensors defined in the framework of finite transformation were also used. To quantify volume variations, it is convenient to use the logarithmic Hencky tensor because its trace equals zero for any incompressible material. This strain mea- surement, very often used in the one-dimensional processing of tensile tests, is classically defined by

$\varepsilon=\ln (\boldsymbol{U})$,

where $\boldsymbol{U}$ is the symmetric and positive definite tensor coming from the polar decomposition of $\boldsymbol{F}$,

$\boldsymbol{F}=\boldsymbol{R} \cdot \boldsymbol{U}$

$\boldsymbol{R}$ being a rotation tensor. As strain-rate measure, we used the time derivative of the Green-Lagrange strain tensor $\boldsymbol{E}$ defined by:

$\boldsymbol{E}=\frac{1}{2}\left(\boldsymbol{F}^{T} \cdot \boldsymbol{F}-\mathbf{1}\right)$

\subsection{A heterogeneous tensile test}

Fig. 1 shows the time evolution of the longitudinal distribution of heat sources $w_{\mathrm{ch}}^{\bullet}(x, 0, t)$ while Fig. 2 shows, in the same way, the profile of the longitudinal strain-rate component $\dot{E}_{x x}(x, 0, t)$. Although results were not obtained on a same sample, the calorimetric and kinematic fields show strong similarities:

- the softening part of the conventional stress-strain curve corresponds, at the same time, with a progressive concentration of heat sources and with a strain localization characterized by high strain-rates;

- these localization mechanisms lead to the inception of a neck which then spreads regularly throughout the test section. The image processing shows that the material is almost exclusively strained in the necking lips where heat sources of high intensity develop. Note that during the expansion of the necking region, the conventional stress remains approximately constant, even when one of both lips is stopped in the connection zone of the sample.

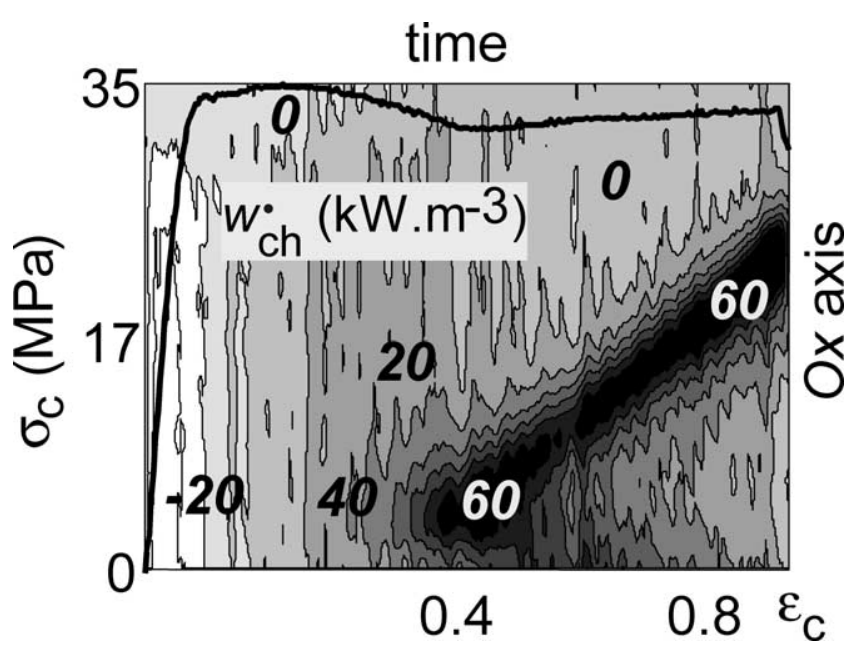

Fig. 1. Time evolution of the longitudinal heat source profile $w_{\mathrm{ch}}^{\bullet}(x, 0, t)$. The test is velocity-controlled $=0.05 \mathrm{~mm} \cdot \mathrm{s}^{-1}$ ). 


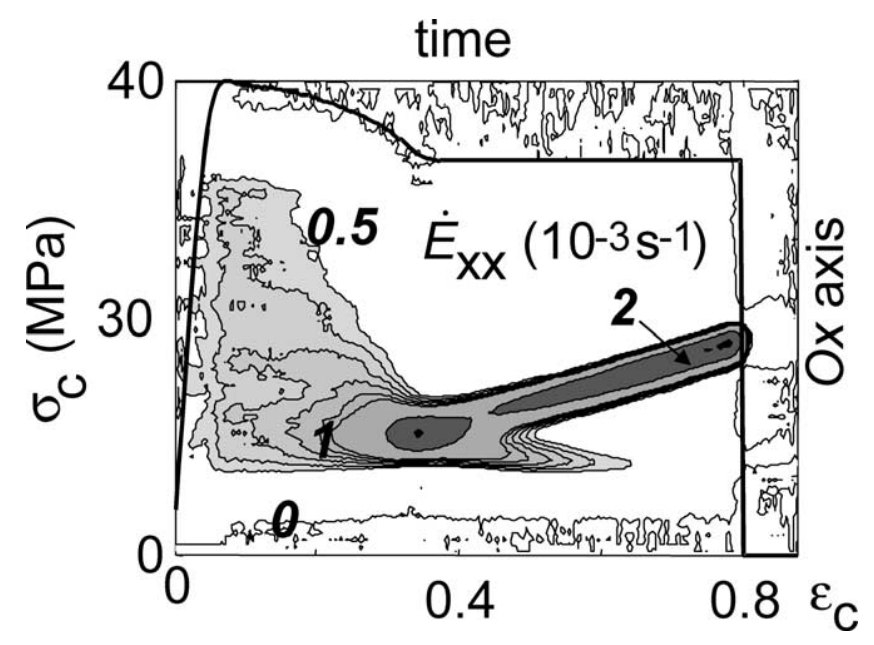

Fig. 2. Time evolution of the longitudinal strain-rate profile $\dot{E}_{x x}(x, 0, t)$. The test is velocity-controlled $=0.05 \mathrm{~mm} \cdot \mathrm{s}^{-1}$ ).

\subsection{A local construction of stress-strain diagram}

When necking appears, it is legitimate to wonder about the uniformity of the stress distribution within the gauge part of the sample. An analysis based on the properties of the strain fields was developed in [14] to construct a complete stress pattern by integrating the equilibrium equations. In this work, the plane-stress hypothesis for thin flat samples was adopted, assuming that the tensile stress distribution remains uniform within each cross-section of the gauge part. Consequently, the mass balance and the incompressibility hypothesis lead to:

$\sigma_{x x}(x, t)=\sigma_{\mathrm{c}}(t) \cdot \exp \left(\varepsilon_{x x}(x, t)\right)$

where $\varepsilon_{x x}(x, t)$ is the longitudinal Hencky strain. Remember that speckle image processing gave weak strain gradients in the width-direction, hence the $y$-independence of $\varepsilon_{x x}$.

Fig. 3 shows the time-evolution of the longitudinal Cauchy stress profile $\sigma_{x x}(x, t)$. In accordance with Eq. (11), a progressive development of the stress concentration occured during the softening part of the conventional stressstrain curve. Then, the expansion of the necking region gave rise to important stress gradients in the necking lips.

It is interesting to illustrate the mechanical behavior of the material by plotting correspondences between Cauchy stress and Hencky strain. Fig. 4 shows the responses $\left(\sigma_{x x}, \varepsilon_{x x}\right)$ for five for cross-sections. The virual extensometers drawn on the sketch of the sample spot the location of the chosen cross-sections.

In this figure, we first notice that the stress-strain curves are not the same from one section to another. Necking inception takes place in section $\boldsymbol{A}$. The expansion of the necking region is then due to the propagation of two lips. The upper one is rapidly blocked in the connection zone of the sample.

The stress-strain curve at $\boldsymbol{A}$ presents a positive hardening modulus ( $\left.\mathrm{d} \sigma_{x x} / \mathrm{d} \varepsilon_{x x}>0\right)$ and, after a "yield" stress, a positive curvature $\left(\mathrm{d}^{2} \sigma_{x x} / \mathrm{d} \varepsilon_{x x}^{2}>0\right)$. This exponential hard-

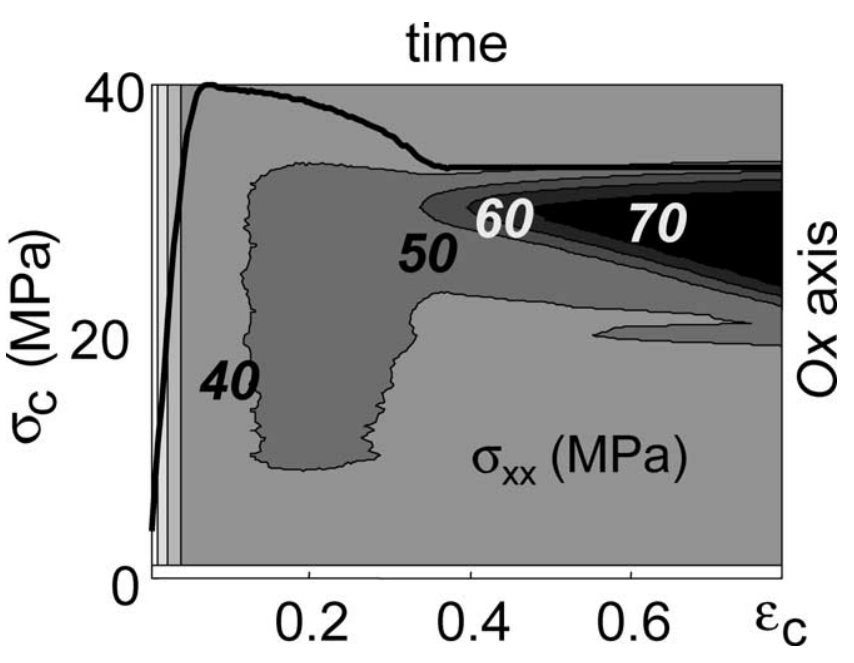

Fig. 3. Time evolution of the tensile stress profile $\sigma_{x x}(x, 0, t)$. The test is velocity-controlled $=0.05 \mathrm{~mm} \cdot \mathrm{s}^{-1}$ ).

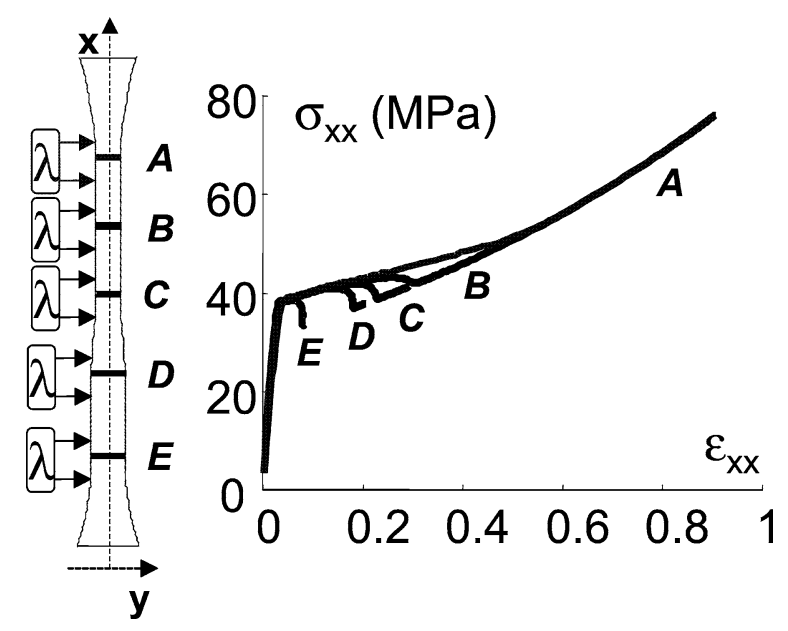

Fig. 4. Local stress-strain curves $\left(\sigma_{x x}, \varepsilon_{x x}\right)$ for five cross-sections regularly chosen in the sample gauge part.

ening may explain the mechanism of expansion at constant load of the necking region, contrary to some metallic materials where the necking region narrows for positive hardening modulus but negative curvature [14].

For sections $\boldsymbol{B}, \boldsymbol{C}$ and $\boldsymbol{D}$, the stress decreases during the sample softening and remains constant until the second necking lip arrives. For each curve, the turning point indicates the passage of the lip. From then on, the stress increases again and the stress-strain curve joins the one of section $\boldsymbol{A}$ gradually. Until the end of the test, the necking never reached the section $\boldsymbol{E}$ and no turning point occurred.

\section{Concluding comments}

We developed two independent complementary imaging techniques providing whole-field calorimetric and kinematic data during mechanical tests. This information is useful to 
distinguish the material behavior from the structure effects. Indeed, in the particular case of tensile tests on polyamide, the precocity of heterogeneity inception led us to consider the sample as a structure. The image processing showed narrow zones (the necking lips) where strain-rates, heat sources and thus temperature gradients were very important. These lips marked the boundary of the necking region which expanded during the loading. Effects of this expansion mode were also translated by a turning point in the local stressstrain curves.

To go further in the establishment of the local energy balance and in the determination of the constitutive equations, it is now necessary to combine thermal and kinematic data. Indeed, when convective terms are no longer negligible, a proper computation of the particular time derivative requires the knowledge of the temperature distribution and the velocity field. This combination was already tested for metallic materials [15] and should be transposed in a near future to polymers that may overcome very large strains.

\section{References}

[1] P. Germain, Cours de Mécanique des Milieux Continus, Masson, Paris, 1975, pp. 138-200.

[2] P. Germain, Q.S. Nguyen, P. Suquet, Continuum thermodynamics, J. Appl. Mech. 50 (1983) 1010-1020.

[3] P.R. Guduru, A.J. Rosakis, G. Ravichandran, Dynamic shear bands: an investigation using high speed optical and infrared diagnostics, Mech. Materials 33 (2001) 371-402.
[4] P.R. Guduru, G. Ravichandran, A.J. Rosakis, Observations of transient high temperature vortical microstructures in solids during adiabatic shear banding, Phys. Rev. E. 64 (2001) 1-6, 036128.

[5] A.T. Zehnder, P.R. Guduru, A.J. Rosakis, G. Ravichandran, Million frames per second infrared imaging system, Rev. Sci. Instruments 17 (10) (2000) 3762-3768.

[6] Y. Rabin, D. Rittel, Infrared temperature sensing of machanically loaded specimens: thermal analysis, Experimental Mech. 40 (2) (2000) $1-6$.

[7] A. Chrysochoos, F. Belmhajoub, Thermographic analysis of thermomechanical couplings, Arch. Mech. 44 (1) (1992) 55-68.

[8] A. Chrysochoos, H. Louche, An infrared image processing to analyse the calorific effects accompanying strain localization, Internat. J. Engng. Sci. 39 (2000) 1759-1788.

[9] H.H. Bailey, F.W. Blackwell, C.L. Lowery, J.A. Ratkovic, Image correlation: Part I. Simulation and analysis, report prepared for United States Air Force Project RAND, R-2057/1-PR, 1976.

[10] A. Oulamara, G. Tribillon, J. Duvernoy, Subpixel speckle displacement measurement using a digital processing technique, J. Mod. Opt. 35 (1988) 1201-1211.

[11] B. Wattrisse, A. Chrysochoos, J.-M. Muracciole, M. Nemoz-Gaillard, Analysis of strain localization during tensile tests by Digital Image Correlation, Experimental Mech. 41 (1) (2000) 29-38.

[12] H.B. Callen, Thermodynamics and an Introduction to Thermostatistics, Wiley, New York, 1987, pp. 307-324.

[13] D. Kondepoudi, I. Prigogine, Modern Thermodynamics, From Heat Engines to Dissipative Structures, Wiley, New York, 1998, pp. 333404.

[14] B. Wattrisse, A. Chrysochoos, J.-M. Muracciole, M. Nemoz-Gaillard, Kinematic manifestations of localization phenomena in steels by Digital Image Correlation, Eur. J. Mech. A Solids 20 (2001) 189-211.

[15] A. Chrysochoos, J.-M. Muracciole, B. Wattrisse, Experimental analysis of strain and damage localization, in: A. Benallal (Ed.), Symposium on Continuous Damage and Fracture, Cachan, France, 2000, pp. 4151. 